\title{
A high-yield isolation and enrichment strategy for human lung microvascular endothelial cells
}

\author{
Christa Gaskill' and Susan M. Majka ${ }^{1,2}$ \\ 'Department of Medicine, Division of Allergy, Pulmonary and Critical Care Medicine, Vanderbilt University, Nashville, TN, USA; ${ }^{2}$ Vanderbilt Center for Stem Cell \\ Biology, Vanderbilt University, Nashville, TN, USA
}

\begin{abstract}
Vasculopathies, characterized by the formation of fragile and abnormal microvessels, are associated with the severity of many chronic lung diseases, including pulmonary fibrosis, emphysema/chronic obstructive pulmonary disease, systemic sclerosis, and hypertension. However, the study of human lung vasculature has been limited by the ability to isolate generous quantities of microvascular endothelial cells (MVEC) free from mesenchymal contamination. Expansion and passaging of primary human MVEC in vitro typically results in loss of a traditional phenotype in favor of an intermediate mesenchymal one, as early as passage five. Here we provide a detailed protocol for the selection of large quantities of enriched primary human lung MVEC based upon differential adherence from mesenchyme and simple magnetic separation, which decreases the need for excessive passaging, in order to obtain sufficient cell numbers to successfully freeze stock cultures. Additional protocols are provided for Ac-di-LDL selection, characterization, and a sandwich angiogenesis method of functional tube formation. The complete protocol including cell isolation and characterization takes approximately six weeks to complete.
\end{abstract}

\author{
Keywords \\ lung microvascular endothelial cells, primary cells \\ Date received: 27 September 2016; accepted: 5 October 2016 \\ Pulmonary Circulation 2017; 7(I) 108-1 16 \\ DOI: 10.1 I 77/20458932I 7702346
}

\section{Introduction}

The pulmonary microvasculature is the primary site for gas exchange in the lung, facilitating oxygenation of red blood cells and excretion of carbon dioxide. Pathological changes in the pulmonary microvasculature may both precede and/ or exacerbate a number of chronic adult lung diseases, including but not limited to pulmonary fibrosis (PF), chronic obstructive pulmonary disease (COPD), and connective tissue diseases including systemic sclerosis (SSc). Additionally, vascular remodeling may result in pulmonary arterial hypertension (PAH) or pulmonary hypertension $(\mathrm{PH})$ associated with PF, COPD and SSc. Pulmonary vasculopathies, also known as vascular dysfunction or disease (PVD), are characterized by dysfunctional vascular structure and function. PVD is believed to be asymptomatic until severe, when approximately $50-70 \%$ of the pulmonary vascular bed is lost and pulmonary vascular resistance and pulmonary artery pressure rises leading to the clinical presentation and diagnosis of $\mathrm{PH} .{ }^{1}$ Considerable progress has been made in understanding the role that various cell populations, including endothelial cells, smooth muscle cells, and fibroblasts, play in the remodeling that defines vascular dysfunction during disease. However, our current understanding of the cell-based mechanisms are incomplete, demonstrating a need for primary vascular cell types to further study and understand disease processes. To overcome this limitation, we sought to develop a robust method based on the inherently different properties of cell adhesion, between MVEC and lung mesenchymal cells, in order to enrich for primary lung MVEC. Because expansion of

\section{Corresponding author:}

Susan M. Majka, Vanderbilt University, Division of Allergy, Pulmonary and Critical Care Medicine, II6I 2Ist Ave S T-1218 MCN, Nashville, TN 37232, USA.

Email: susan.m.majka@vanderbilt.edu

\section{(c) $(1) \Theta$ Creative Commons Non Commercial CC-BY-NC: This article is distributed under the terms of the Creative Commons Attribution-NonCommercial 3.0 License (http://www.creativecommons.org/licenses/by-nc/3.0/)} which permits non-commercial use, reproduction and distribution of the work without further permission provided the original work is attributed as specified on the SAGE and Open Access pages (https://us.sagepub.com/en-us/nam/open-access-atsage).

\author{
(C) 2017 by Pulmonary Vascular \\ Research Institute. \\ Reprints and permissions: \\ sagepub.co.uk/journalsPermissions.nav \\ journals.sagepub.com/home/pul
}

(SAGE 
primary human MVEC in vitro typically results in loss of phenotype in favor of an intermediate mesenchymal one, we hypothesized that if a substantial number of viable MVEC could be enriched from explanted lung tissue quickly following isolation, a greater number of stock lines could be created. Here we provide a detailed protocol for the selection of enriched primary human lung MVEC, based upon differential adherence and simple magnetic separation, decreasing excessive passaging. Enrichment and lineage specificity was documented by Ac-di-LDL uptake, characterization by flow cytometry, quantitative polymerase chain reaction (qPCR) and immunofluorescent staining as well as a sandwich angiogenesis assay of functional tube formation.

\section{Methods}

\section{Isolation of adherent cell fractions from lung tissue explants}

Human explanted lung tissue was isolated post transplant or autopsy in 1-in. cubes stored in DMEM (Vanderbilt IRB Protocol 9401). The viability and quantity of cells obtained from post-transplant tissue is significantly greater than postautopsy tissue.

Tissue was minced to achieve the consistency of paste in a 100-mm dish, using scissors and disposable scalpels. Minced tissue was then transferred to a solution of Type II collagenase (Worthington sterile filtered; LS004202) in Hank's balanced saline (HBSS; resuspend lyophilized container in approximately $35 \mathrm{~mL}$ to yield $515 \mathrm{U} / \mathrm{mL}$ ). For fibroblast (FB) isolation, tissue is transferred to $23 \mathrm{~mL}$ of collagenase in a 50-mL conical tube (per cube). For the MVEC isolation, tissue is transferred into a total of $90 \mathrm{~mL}$ of collagenase and then divided between three $50 \mathrm{~mL}$ conical tubes. The tissue is then digested for $2.5 \mathrm{~h}$ with agitation (rotating hybridization oven) at $37^{\circ} \mathrm{C}$.

Following digest, an equal volume of HBSS was added per tube followed by trituration with a $10-\mathrm{mL}$ pipet. The solution was centrifuged to pellet cells and tissue for $10 \mathrm{~min}$ at $1500 \mathrm{rpm}$. The FB digest was plated in two T75s with $\alpha$ MEM, 20\% fetal bovine serum (FBS) with pen/ strep and fungizone (standard MSC/MPC medium). ${ }^{2-4}$ The MVEC preparation was plated in three T75s coated with attachment factor (AF) (Life Technologies S-006-100; gelatin) with MVEC media (LONZA; CC-3156 \& CC-4147 or CC-3202); 16-20 h later the media was changed to remove debris. At $24 \mathrm{~h}$, post-plating, additional heparin $(0.75 \mathrm{U} / \mathrm{mL})$ was added to the complete LONZA medium, in order to decrease $\mathrm{FB} /$ mesenchymal growth. Within $48-72 \mathrm{~h}$, the cells spread and assumed characteristic phenotypes. Heparin was added only after the cells had adhered because it can decrease attachment.

To passage the MVEC cell prep, flasks were washed with DPBS followed by the application of Versene (Gibco 15040066). Flasks were place in the incubator for $1 \mathrm{~min}$ at a time and subsequently viewed to identify that $100 \%$ of the cells had lifted. The lifted cells were plated onto AF coated flasks (passage 1). When flasks were nearly confluent (day 12 post isolation), cells were lifted with Versene and magnetic bead separation was performed using CD31/PECAM, according to the manufacturer's instructions (Miltenyi kit no 130-091-935; passage 2), with the following exception: use PBS $+2 \%$ FBS as buffer and omit the kit blocking instructions. Two million cells were separated on the column. Upon subsequent passages, we performed a quick first pass lift with Versene, in order to deplete FB, further enriching for MVEC (which remain adherent). The flasks were viewed closely and gently tapped to remove FB. Trypsin was applied to quickly lift and pass the remaining enriched MVEC. Further enrichment may be performed by incubating 50-75\% confluent MVEC in Ac-di-LDL-Alexa 488 (Life Technologies L23380) at $10 \mathrm{~g} / \mathrm{mL}$ in growth media. Flow cytometry may be used to sort out the cells with the highest peak fluorescence. FB passaging was performed using trypsin and standard protocols. MVEC were always cultured on AF coated flasks.

\section{Acetyl-di-LDL uptake}

MVEC cells approximately $75 \%$ confluent $(3,000,000$ at this stage; passage 4 , day 31 ) were incubated with $5-10 \mathrm{ug} / \mathrm{mL}$ acetylated (Ac-di LDL-Alexa 488 (Life Technologies L23380) in growth medium for $3 \mathrm{~h}^{5}$ Known positive and negative controls were also prepared to set sorting gates. In this case, pulmonary artery endothelial cells (PAEC; LONZA CC-2530) were used as a positive and lung FB as a negative control.

Ac-di-LDL was then removed and cell rinsed once with HBSS and trypsinized for approximately 2 min using standard protocols. Four volumes of complete media were immediately added to inhibit the trypsin. FACS was employed to sort and collect on the top $50 \%$ or more positive fluorescent fraction based on the fluorescence intensity of positive and negative control cells. FB were used as a negative control for Ac-di-LDL uptake to set the selection gates. Using the BD FACSAria III (BD Biosciences, San Jose, CA, USA) the recommended wavelengths used for cell sorting included: excitation $=488 \mathrm{~nm}$, emission $=550 \mathrm{~nm}$. Sorted cells were collected into complete growth medium and re-plated in AF coated T25 flasks. Additional heparin is unnecessary for maintenance of these cultures at this stage.

\section{MVEC characterization}

Flow cytometry was used to characterize cell surface determinant expression by MVEC using standard protocols. ${ }^{3-6}$ The cells were stained with antibodies to detect the markers presented in Table 1 using a BD FACSAria III (BD Biosciences, San Jose, CA, USA). Fluorescent minus one (FMO) and isotype (eBioscience, San Diego, CA, USA) controls were used to set the gates. DAPI was used to exclude dead cells. 
Table I. Reagents.

\begin{tabular}{|c|c|c|c|}
\hline Antibodies & & Vendor & Ref no. \\
\hline CDI44 & PE & ebio & $12-1449-80$ \\
\hline CDI05 & PE & ebio & $|2-| 057-4 \mid$ \\
\hline CDI06 & PE & Ebio & $|2-| 069-4 \mid$ \\
\hline CD338 & PE & $\mathrm{BD}$ & 561180 \\
\hline B220 & PE & ebio & 12-0452-82 \\
\hline CD3I & PE & $\mathrm{BD}$ & 555446 \\
\hline CDI46 & FITC & ebio & ||$-|469-4|$ \\
\hline CD34 & FITC & ebio & II-0349-42 \\
\hline CDI4 & FITC & ebio & ||$-0 \mid 49-42$ \\
\hline CD44 & FITC & ebio & ||$-044 \mid-82$ \\
\hline CD45 & APC & ebio & $17-9459-42$ \\
\hline CD73 & APC & ebio & $|7-0739-4|$ \\
\hline Isotype & APC & $\mathrm{BD}$ & 555745 \\
\hline CDIO2 & FITC & biolegend & 328507 \\
\hline Human PCR primers & & $5^{\prime}$ to $3^{\prime}$ & \\
\hline \multirow[t]{2}{*}{ CDH5 (VE Cadherin) } & $\mathrm{Fw}$ & TTGGAACCAGATGCACATTGAT & \\
\hline & $\mathrm{Rv}$ & TCTTGCGACTCACGCTTGAC & \\
\hline \multirow[t]{2}{*}{ PECAM } & Fw & CCAAGGTGGGATCGTGAGG & \\
\hline & $\mathrm{Rv}$ & TCGGAAGGATAAAACGCGGTC & \\
\hline \multirow[t]{2}{*}{ VWF } & Fw & CCGATGCAGCCTTTTCGGA & \\
\hline & $\mathrm{Rv}$ & TCCCCAAGATACACGGAGAGG & \\
\hline \multirow[t]{2}{*}{ Tie2 } & Fw & TTAGCCAGCTTAGTTCTCTGTGG & \\
\hline & $\mathrm{Rv}$ & AGCATCAGATACAAGAGGTAGGG & \\
\hline \multirow[t]{2}{*}{ ACTA2 } & Fw & CCTTTGGCTTGGCTTGTCAG & \\
\hline & $\operatorname{Rv}$ & GGTGCGGACAGGAATTGAAG & \\
\hline \multirow[t]{2}{*}{ FLK (VEGFR2) } & Fw & GGCCCAATAATCAGAGTGGCA & \\
\hline & $\mathrm{Rv}$ & CCAGTGTCATTTCCGATCACTTT & \\
\hline \multirow[t]{2}{*}{ FLTI } & Fw & TTTGCCTGAAATGGTGAGTAAGG & \\
\hline & $\operatorname{Rv}$ & TGGTTTGCTTGAGCTGTGTTC & \\
\hline \multirow[t]{2}{*}{ PDGFRB } & Fw & GATGCTGAGGTCCAGCTCTC & \\
\hline & $\operatorname{Rv}$ & CAGGGTGGCTCTCACTTAGC & \\
\hline CSPG4 & & Applied Biosystems & Hs0036|54 \\
\hline
\end{tabular}

The compensation controls were established as cells only, cells + DAPI, cells + APC antibody, cells + PE antibody, cells + FITC antibody, alternatively comp beads were used. The gating strategy routinely included forward scatter/side scatter (FSC)/SSc, single cells gated by SSCWidth/SSC-Height (SSC-W/SSC-H), FSC-W/FSC-H, DAPI to gate out dead cells. A summary of antibodies is presented in Table 1. Known positive and negative controls were also prepared to set sorting gates. In this case, PAEC (LONZA CC-2530) were used as a positive control and lung FB as a negative control. qPCR and immunofluorescent staining were performed to demonstrate the relative expression of endothelial marker transcript and protein using standard methods. ${ }^{2,5,7}$ A summary of PCR primers is presented in Table 1.

\section{Tube-forming assay: sandwich method}

Tissue culture treated two-chamber slides (Lab-Tek 154852) were pre-chilled overnight and coated with 400 microliters diluted Matrigel (Corning 354264, San Jose, CA, USA). For these studies, high concentration Matrigel was diluted 1:1 with ice-cold growth media before use. Matrigel was allowed to polymerize at $37^{\circ} \mathrm{C}$ for $45 \mathrm{~min}$. Concurrently, cells from all lines were trypsinized, filtered, and centrifuged to be resuspended at $1 \times 10^{5}$ cells $/ \mathrm{mL}$ in growth media. A preparation was made by further diluting our Matrigel working stock to $4 \%$ in ice-cold growth media. Cells were mixed in equal volume with the low concentration Matrigel mixture to achieve a final concentration of $5 \times 10^{4}$ cells $/ \mathrm{mL}$ in $2 \%$ Matrigel. A total of 750 microliters was applied to 

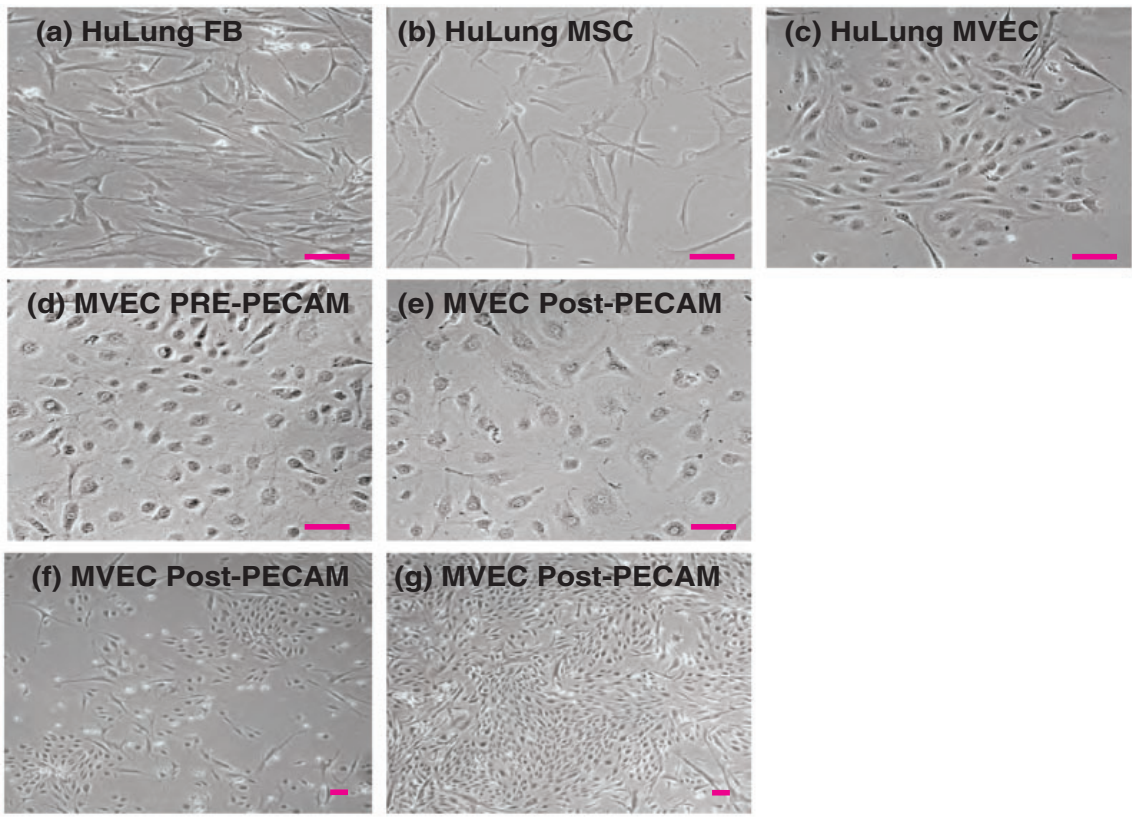

Fig. I Pulmonary MVEC, FB, and MSC phenotypes. Phase contrast images are presented documenting the phenotypes of established primary lung FB, MSC, and MVEC cell lines $(a-c)$ as well as MVEC colonies at various stages of the isolation protocol (d-g). (d) Representative MVEC colony pre-column enrichment. (e) Representative MVEC colony post-column enrichment. (f) Representative MVEC colonies post-column enrichment subconfluent. (g) Representative MVEC colonies post-column enrichment confluent. Scale bar $=25$ micrometers.

each chamber. Slides were incubated at $37^{\circ} \mathrm{C} 5 \% \mathrm{CO} 2$ and tube formation was photographed starting at $30 \mathrm{~min}$ after plating to $4 \mathrm{~h}$ when a significant degree of tube formation had occurred. In all experiments, FB were cultured using $20 \%$ FBS $\alpha$ MEM (Life Technologies 12571-063) while endothelial cells (PAEC and MVEC) were cultured in EBM-2 with EGM-2 MV SingleQuots (Lonza CC-3156, CC-4147).

\section{Results}

\section{Pulmonary MVEC or FB isolation and morphology}

Primary cell isolation and characterization were performed to obtain primary MVEC and FB from lung tissue explants (Fig. 1a and c). Lung mesenchymal stem cells (MSC) were also isolated from FB as previously described ${ }^{2,3}$ (Fig. 1b). The identification of optimal conditions to obtain single cell suspensions from fresh distal lung tissue allowed the initial selection between the two cell types. A higher yield of MVEC was obtained with the increased ratio of collagenase to tissue relative to that of lung FB. Second, plating cells on a gelatin substrate, at a density to allow for colony formation following adherence, aided in the identification and further enrichment of MVEC. Purification was performed exploiting their expression of PECAM1/CD31. Primary CD31 antibody conjugated to a magnetic bead was used in combination with a magnetic column. CD31 expressing MVEC was positively selected (Fig. 1e-g). MVEC colonies comprising 50 or more cells appeared more resistant to detachment during Versene treatment, while the mesenchymal cells readily detached (Fig. 1d). Removal of the mesenchymal cells at this stage allowed for expansion of a highly enriched population of MVEC. The addition of heparin also attenuated residual mesenchymal proliferation, likely through modulation of growth factor signaling. ${ }^{8}$

\section{Analysis and enrichment of human lung MVEC by Ac-di-LDL uptake}

To analyze the relative purity of MVEC and perform a further enrichment step, Ac-di-LDL uptake was used. Di LDL has been utilized for over 20 years to purify EC in culture from contaminating tissue mesenchymal cells. ${ }^{9}$ Lung cells were incubated with fluorescent labeled Ac-di-LDL, analyzed, and sorted by flow cytometry (Fig. 2). Primary lung FB were used as a negative control to set the gates, distinguishing between positive and negative populations. The lung FB demonstrated less than $0.5 \%$ positive uptake of the Ac-di-LDL. There was a clear separation between the negative and positive populations of MVEC, which were 93.0\% positive for Ac-di-LDL uptake. Starting with $3,000,000$ cells, approximately 1,850,000 cells were identified as positive and appropriate for sorting. Enrichment of the MVEC population at this stage may only be necessary for specific analyses. The purity of the sorted population was $100 \%$ (Fig. 2). Ac-di-LDL uptake distinguishes the MVEC from lung mesenchymal FB. 

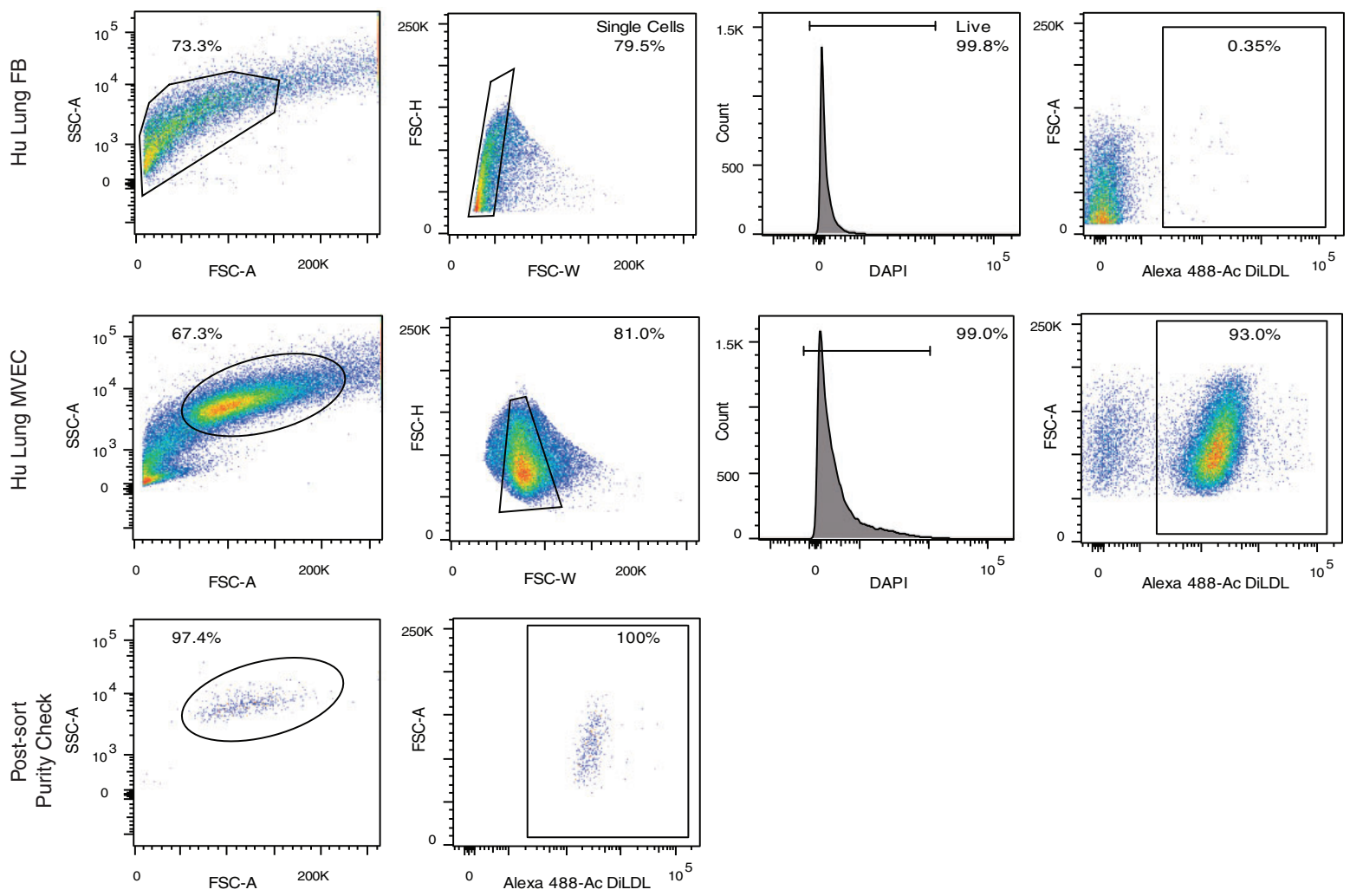

Fig. 2 Analysis and enrichment of human lung MVEC by Ac-di-LDL uptake. Lung cells were incubated with Ac-di-LDL labeled with Alexa 488 and analyzed by flow cytometry. Representative dot plots of the gating strategy are presented. Human lung FB were used as a known negative control for uptake and DAPI was used to exclude dead cells. A post sort purity check demonstrates the purity of the sorted MVEC population.

\section{Lung MVEC expression of endothelial lineage cell surface markers distinct from the tissue mesenchyme}

Lung cells were incubated with primary antibodies directly conjugated to fluorophores and staining intensity analyzed by flow cytometry to quantitate their expression of the endothelial lineage markers, CD144/VE-cadherin, CD31/ PECAM1, CD102/ICAM2, CD105/Endoglin, CD73, CD146/MCAM, CD34, and CD44 (Fig. 3). PAEC were used as a positive control, FB and lung MSC as negative controls. All cell lines were negative for the expression of hematopoietic markers CD45, B220, and CD14. The EC universally expressed CD144, CD31, CD102, CD105, CD73, CD146 and were negative for CD338 (ABCG2). The MVEC heterogeneously expressed CD34 (24\%), CD106 (0.58\%), and CD44 (93\%). Lung FB heterogeneously expressed CD106 (1.65\%) and CD338 (3.2\%) and were negative for the EC markers CD144, CD31, and CD102. The MVEC and PAEC overlapped in their cell surface expression of all the markers with the exception of CD44 (PAEC 73\%; MVEC 93\%). The MVEC were clearly distinct from the FB and MSC, overlapping only in their expression of CD105, CD73, and CD44. Interestingly CD146, 73, 44, and 105 are also considered MSC markers and are routinely used in the panel to define MSC. ${ }^{13}$

\section{Transcriptional and intracellular expression of endothelial markers by primary lung MVEC}

To further define the endothelial characteristics of the primary lung MVEC and distinguish them as distinct from the tissue resident mesenchymal fractions, we performed qPCR analyses using human lung MVEC, PAEC, FB, and MSC. Relative levels of gene expression were quantitated for endothelial lineage markers VECAD, PECAM1, VWF, TIE2(TEK), the vascular endothelial growth factor receptors, FLT1 and FLK, and the pericyte/mesenchymal lineage marker, $P D G F R \beta$ (Fig. 4a). ${ }^{10-12}$ MVEC expressed relatively high levels of all the EC markers and were negative for $P D G F R \beta$. Lung MSC expressed high levels of $P D G F R \beta$ and low levels of Flt1. Immunostaining was also performed to detect and localize Factor VIII or VE-cadherin in isolated primary lung MVEC. As expected, Factor VIII localized to inclusion bodies in the cytoplasm of the cells while VE-cadherin localized to the tight junctions between the MVEC as well as the cytoplasm (Fig. 4b).

\section{MVEC demonstrate tube-forming ability in vitro}

A Matrigel sandwich method was used to analyze functional tube-forming ability of the primary lung MVEC. PAEC were 


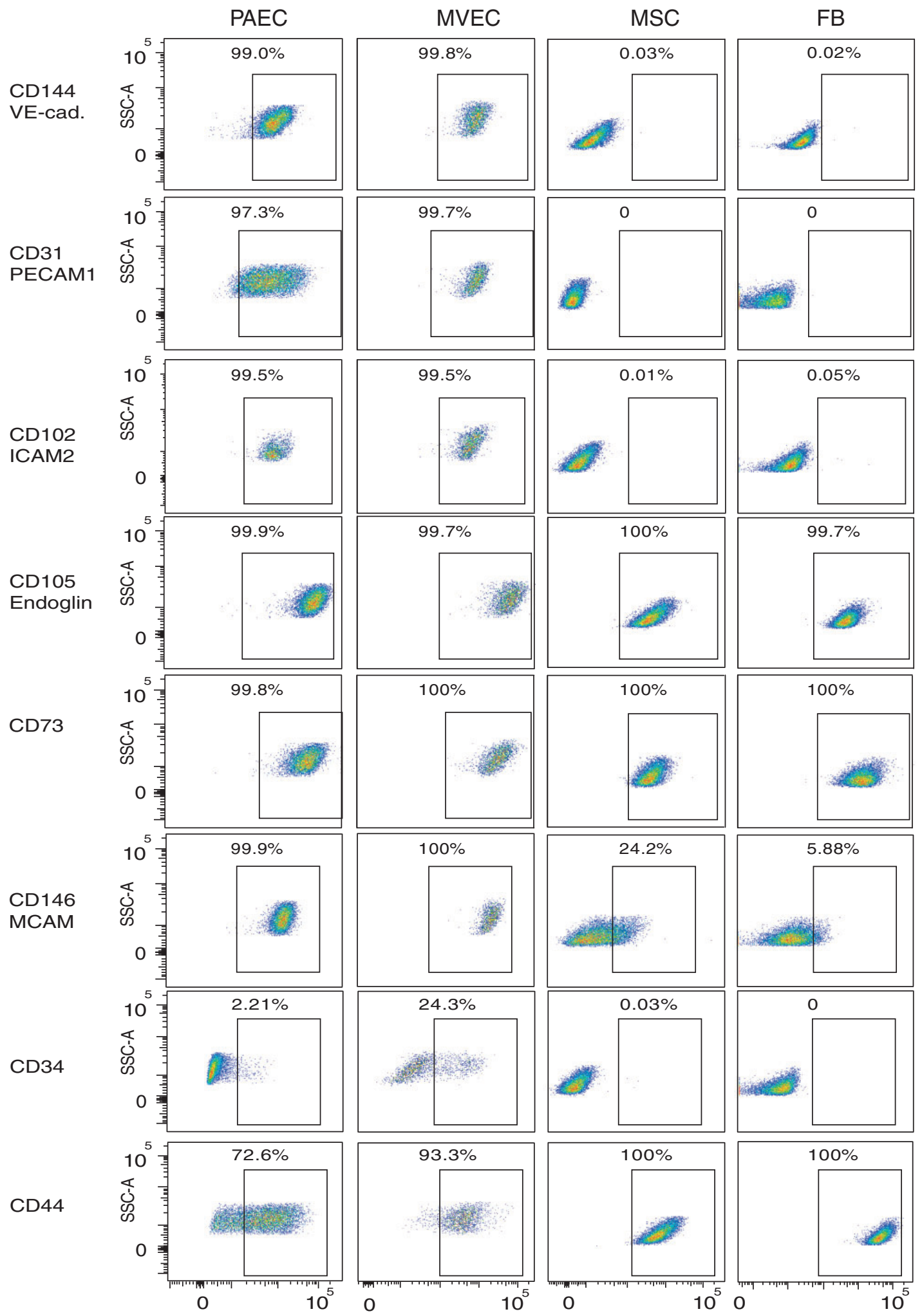

Fig. 3 Expression of endothelial cell surface determinants by primary lung MVEC. Lung cells were incubated with primary antibodies directly conjugated to fluorophores and staining intensity analyzed by flow cytometry. Representative dot plots are presented. Human lung FB and MSC were used as known negative controls, and PAEC as a known positive control. DAPI was used to exclude dead cells. 

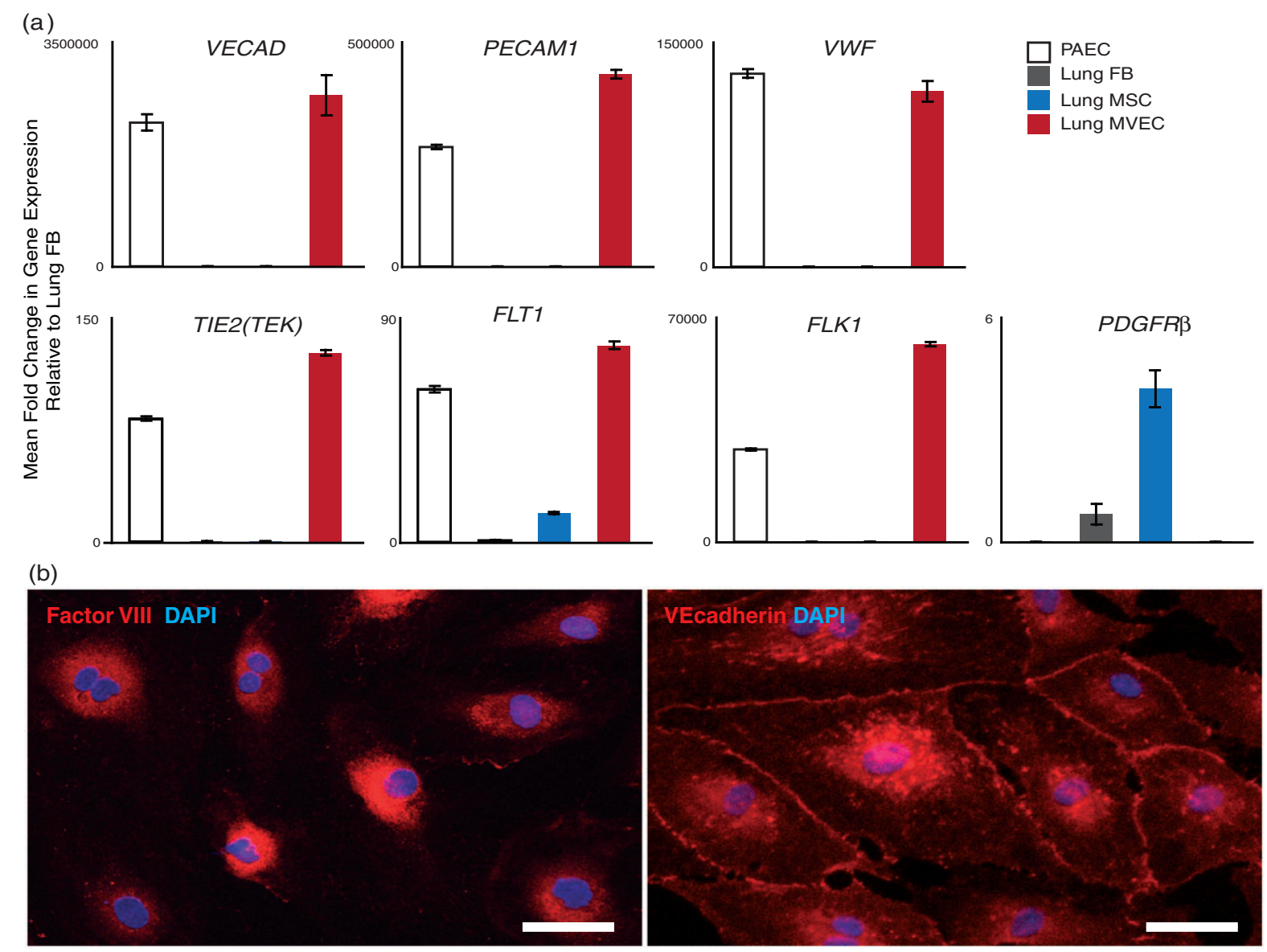

(b)

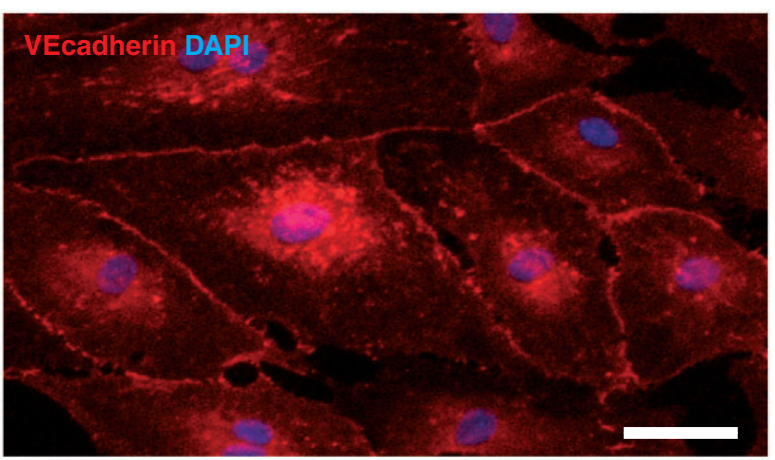

Fig. 4 Transcript and protein expression of endothelial markers by primary lung MVEC. (a) qPCR analyses of human lung MVEC, PAEC, FB, and MSC was performed to quantitate relative levels of gene expression for endothelial lineage markers and the pericyte/mesenchymal lineage marker, PDGFR ${ }^{\circledR}$. (b) Immunostaining was performed to localize Factor VIII and VE-cadherin in isolated primary lung MVEC (red, DAPI stained nuclei blue). Scale bar $=25$ micrometers.

used as a positive control and primary lung $\mathrm{FB}$ as a negative control. At $4 \mathrm{~h}$, both PAEC and primary lung MVEC formed characteristic angiogenic tube structures (Fig. 5). In contrast, lung FB formed clusters or cord-like structures.

\section{Discussion}

Obtaining reasonable yield of primary human lung MVEC from tissue explants free from mesenchymal contamination at low passage, and with traditional morphology and endothelial characteristics, has posed a significant challenge. This limitation coupled to the availability of tissue has resulted in studies of the pulmonary microvasculature being conducted with small numbers of patient primary cell lines (n number), variable experimental outcomes due to variation in cell phenotypes and purity, as well as the use of animal cells for a majority of studies. To address our hypothesis, we evaluated the use of positive selection of MVEC based on differential adherence characteristics distinct from tissue mesenchymal cells, specifically FB. Using a protocol for the selection of enriched primary human lung MVEC, based upon differential adherence and simple magnetic separation, we demonstrated enrichment and lineage specificity by Ac-di-LDL uptake, characterization by flow cytometry, qPCR, and immunofluorescent staining as well as functional angiogenic tube formation. This protocol is scalable and may be used to obtain a large number of enriched control and disease patient lung MVEC. These reported techniques expand upon recently published protocols for the isolation of pulmonary MVEC. ${ }^{14-16}$ The use of magnetic bead separation of MVEC is similar to the protocol previously published by Mackay et al. ${ }^{16}$ in which positive bead selection was used to achieve 1-9 million MVEC by passages 4-6 from $50 \mathrm{~g}$ of lung tissue, with the reported purity in the range of approximately $77-90 \%$. Here we achieved 3 million cells, at low passage, from a small section of explanted lung, approximately $3 \mathrm{~g}$.

Significant variations in the protocols that may have increased our yield included the adherence step we utilized following tissue digest, as well as the use of supplemental heparin to decrease mesenchymal proliferation. The adherence step facilitated the removal of tissue debris and dead cells versus the use of a 100 micrometer filter, which likely removes the clusters of MVEC that should appear as 

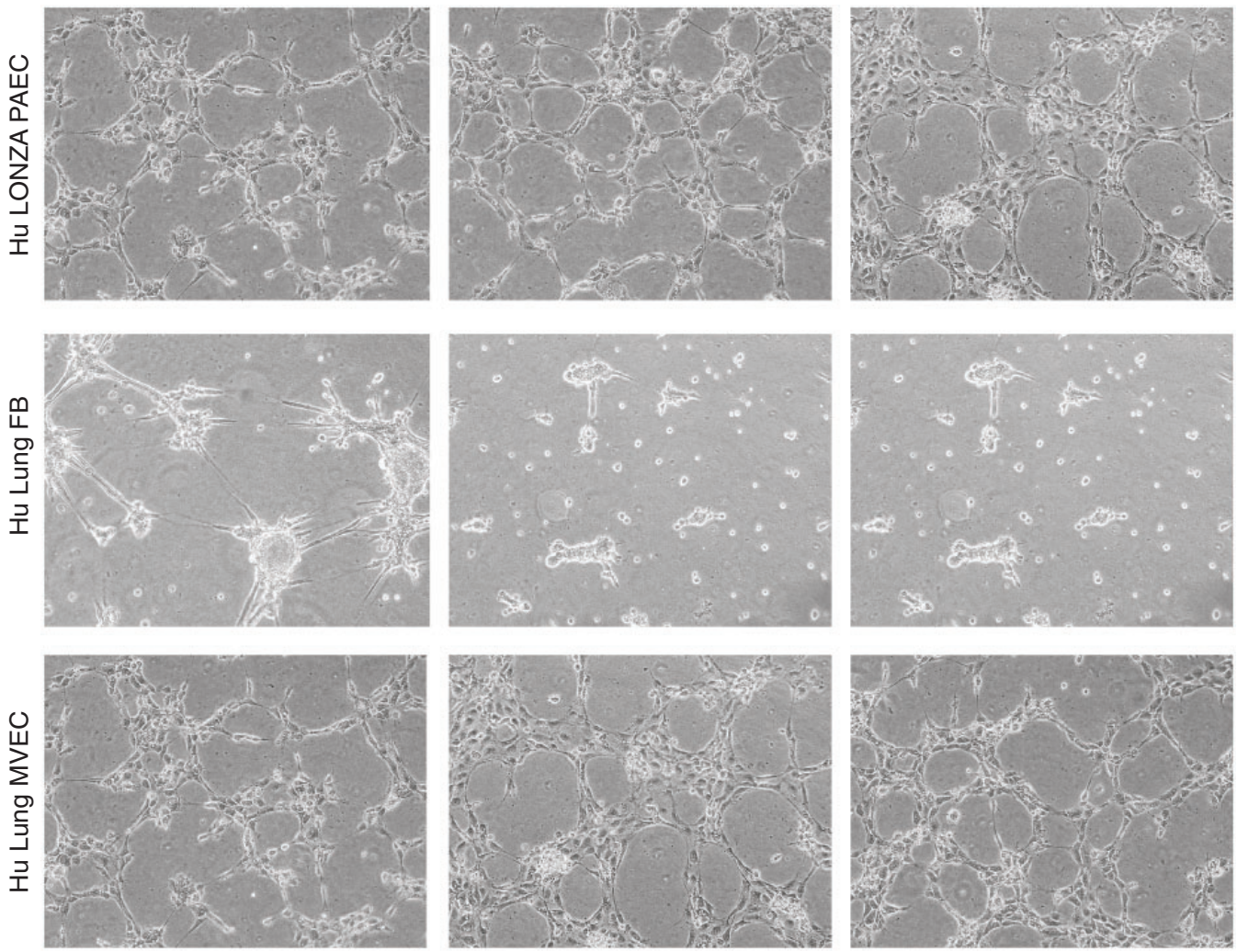

Fig. 5 Demonstration of MVEC tube-forming ability in vitro. A Matrigel sandwich method was used to analyze tube-forming ability of the primary lung MVEC. PAEC were used as a positive control and primary lung FB as a negative control. Phase contrast images are presented documenting the formation of angiogenic tube structures at $4 \mathrm{~h}$ for PAEC and primary lung MVEC. Lung FB formed clusters or cord-like structures.

"beads on a string" following digest due to their tight cellcell contacts and basement membrane. The MVEC cultures were also plated to achieve less than $30 \%$ confluence, in order to allow colonies to expand in size. The second published protocol utilized the clustering of MVEC following digest to select them using a filtration step. Comhair et al. ${ }^{14}$ digested lung tissue explants for a brief interval of $20 \mathrm{~min}$, then, using applied pressure, forced clusters of MVEC from the tissue. Isolated cells were filtered and EC clusters rinsed from the top of the filter and plated on fibronectin-coated plastic.

The investigators also used supplemental heparin to control contaminating mesenchymal growth. However, it was unclear how the contaminants were removed, what yield or purity was achieved, as only isotype antibodies were used as controls for flow cytometric characterization. Generalized isolation of MVEC from tissue was also reported by van Beijnum et al. ${ }^{17}$ using either magnetic separation or flow cytometry enriching by CD31, CD34, CD146, or CD105.

Our data demonstrate that CD34 expression is highly variable and CD146 and CD105 are not specific to MVEC. As expected CD31, VE-cadherin and ICAM2 were expressed by EC, and not lung mesenchymal cells, $\mathrm{FB}$, or MSC. Following digest of $1 \mathrm{~g}$ of tissue the investigators could identify and sort approximately 1,000,000 cells with $90 \%$ purity; however, their yield of viable cells was not reported.

The most significant findings from this study comparing the primary human MVEC with lung FB and MSC was that the MVEC colonies maintained their adherence and contacts during brief Versene treatment, which allowed for their further selection and expansion, without passaging and separating them. The culture of MVEC colonies, versus independent cells after passaging, may facilitate the maintenance of their morphology, characterized by tight junctions and basement membrane. The maintenance of the MVEC cell-cell contacts during isolation is also the underlying reason that isolating primary EC from tissue digests by flow cytometry, which inherently requires single cell suspensions, typically results in a low yield of viable cells. An additional concern with flow cytometric isolation of MVEC is the damage to the cell membranes necessary to obtain a single cell suspension. In contrast, circulating EC or EC progenitors that may be successfully isolated using flow cytometry. ${ }^{18}$

Taken together our results demonstrate that this protocol, based on selective adhesion, efficiently enriches for human lung MVEC. Thus, building upon existing protocols and providing a straightforward method for rapid and higher yield isolation of MVEC from explanted lung 
tissue. This higher yield of lower passage cells should serve as a resource for many investigators interested in preparing MVEC stock for the study of pulmonary microvasculature.

\section{Acknowledgments}

The authors thank Jonathan Kropski, M.D. and Joshua Fessel M.D., Ph.D, for their provision of tissue explants as well as the expert technical assistance provided by Catherine E. Alford and the Research Flow Cytometry Laboratory at the Nashville VA Medical Center and Karen Helm in the University of Colorado Cancer Center's Flow Cytometry Shared Resource (P30CA046934 NCI).

\section{Conflict of interest}

The author(s) declare that there is no conflict of interest.

\section{Funding}

This work was funded by grants to S.M. Majka from the NIH R01HL091105 and NIH R01HL11659701. Additional funding was also provided by PPG-5P01HL108800-04 (PI: J. Loyd) and PPG-2 P01 HL 092870-06 (PI: T. Blackwell). The project was supported in part by the National Center for Research Resources, Grant UL1 RR024975-01, and is now at the National Center for Advancing Translational Sciences, Grant 2 UL1 TR000445-06.

\section{References}

1. Austin ED, Kawut SM, Gladwin MT, et al. Pulmonary hypertension: NHLBI Workshop on the Primary Prevention of Chronic Lung Diseases. Ann Am Thorac Soc 2014; 11(Suppl. 3): S178-S185.

2. Gaskill C, Marriott S, Pratap S, et al. Shared gene expression patterns in mesenchymal progenitors derived from lung and epidermis in PAH: Identifying key pathways in pulmonary vascular disease. Pulm Circ 2016; 6(4): 483-497.

3. Marriott S, Baskir RS, Gaskill C, et al. ABCG2(pos) lung mesenchymal stem cells are a novel pericyte subpopulation that contributes to fibrotic remodeling. Am J Physiol Cell Physiol 2014; 307(8): C684-C698.

4. Martin J, Helm K, Ruegg P, et al. Adult lung side population cells have mesenchymal stem cell potential. Cytotherapy 2008; 10(2): 140-151.
5. Chow K, Fessel JP, Kaorilhida S, et al. Dysfunctional resident lung mesenchymal stem cells contribute to pulmonary microvascular remodeling. Pulm Circ 2013; 3(1): 31-49.

6. Alvarez DF, Helm K, DeGregori J, et al. Publishing flow cytometry data. Am J Physiol Lung Cell Mol Physiol 2010; 298(2): L127.

7. West JD, Austin ED, Gaskill C, et al. Identification of a common Wnt-associated genetic signature across multiple cell types in pulmonary arterial hypertension. Am J Physiol Cell Physiol 2014; 307(5): C415-C430.

8. Kendall RT and Feghali-Bostwick CA. Fibroblasts in fibrosis: novel roles and mediators. Front Pharmacol 2014; 5: 123.

9. Voyta JC, Via DP, Butterfield CE, et al. Identification and isolation of endothelial cells based on their increased uptake of acetylated-low density lipoprotein. J Cell Biol 1984; 99(6): 2034-2040.

10. Armulik A, Abramsson A and Betsholtz C. Endothelial/pericyte interactions. Circ Res 2005; 97(6): 512-523.

11. Birbrair A, Zhang T, Files D, et al. Type- 1 pericytes accumulate after tissue injury and produce collagen in an organ-dependent manner. Stem Cell Res Ther 2014; 5(6): 122.

12. Paquet-Fifield S, Schluter H, Li A, et al. A role for pericytes as microenvironmental regulators of human skin tissue regeneration. J Clin Invest 2009; 119(9): 2795-2806.

13. Dominici M, Le Blanc K, Mueller I, et al. Minimal criteria for defining multipotent mesenchymal stromal cells. The International Society for Cellular Therapy position statement. Cytotherapy 2006; 8(4): 315-317.

14. Comhair SAA, Xu W, Mavrakis L, et al. Human primary lung endothelial cells in culture. Am J Respir Cell Mol Biol 2012; 46(6): 723-730.

15. Bryant AJ, Carrick RP, McConaha ME, et al. Endothelial HIF signaling regulates pulmonary fibrosis associated pulmonary hypertension. Am J Physiol Lung Cell Mol Physiol 2016; 310(3): L249-262.

16. Mackay LS, Dodd S, Dougall IG, et al. Isolation and characterisation of human pulmonary microvascular endothelial cells from patients with severe emphysema. Respir Res 2013; 14(1): 1-11.

17. van Beijnum JR, Rousch M, Castermans K, et al. Isolation of endothelial cells from fresh tissues. Nat Protocols 2008; 3(6): 1085-1091.

18. Khan SS, Solomon MA and McCoy JP. Detection of circulating endothelial cells and endothelial progenitor cells by flow cytometry. Cytometry B Clin Cytom 2005; 64B(1): 1-8. 